\title{
Research on Attitude Control Algorithm of PID Quadrotor based on LPV Method
}

\author{
Chenyu Hu${ }^{1,}$ a , Hui Yang1, b \\ School of Air Transportation, Shanghai University of Engineering Science, Shanghai 201620, China; \\ ayuri_chen_28@163.com, byanghui@nuaa.edu.cn
}

Keywords: Quadrotor, Attitude Controler, LPV Method, PID Controler.

\begin{abstract}
The quadrotor is a non-linear, multivariable, strongly coupled under actuated system that is very difficult to control precisely. In this paper, the mathematic model of the quadrotor is established. Since the mathematical model is nonlinear, the model used in the controller and the simulation analysis is often linear. Therefore, it is necessary to linearize the nonlinear model first. Using LPV method, and then use the PID algorithm to control the quadrotor attitude. Simulink is used to simulate the model, which proves that PID has significant effect on attitude control of quadrotor.
\end{abstract}

\section{Introduction}

Quadrotor first appeared in the 20th century, 20 years, the beginning as a military training purposes, and later because of the outstanding performance in the military, quickly attracted the attention of countries around the world. Compared with conventional aircraft, the quadrotor has the advantages of simple structure, light weight, small volume, easy maintenance, low cost, strong flexibility, easy to carry and simple operation, so whether it is in the military field or civilian field, The application prospect of the aircraft is very broad[1].

The quadrotor is a non-coaxial multi-rotor aircraft capable of vertical take-off and landing [2]. It is only necessary to control the flight attitude of the quadrotor by adjusting the rotational speed of the four rotors. Since the quadrotor has four symmetrically distributed rotors, it is possible to counteract the momentary torque of each rotor, so the tail rotor is not needed to make the structure of the quadrotor simpler [3]. The lift of the four rotors is more uniform than the single rotor, and the flight attitude is more stable [4]. The quadrotor has six degrees of freedom, but the controllable adjustment has only four motor speeds, and therefore belongs to a typical non-linear, multivariable, strongly coupled under actuated system [5]. In order to enhance the stability and safety of the aircraft, it is necessary to establish an accurate mathematical model and a well-designed control system. In this paper, the classical PID algorithm is used to control the quadrotor and simulate on Simulink, and a good control effect is obtained.

\section{Mathematical Model Establishment}

Modeling of the quadrotor must solve the problem of the coordinate system, so the establishment of two coordinate system of the ground coordinate system $G$ and the body coordinate system $B$, as shown in Figure 1 below:

Usually the position and attitude of the quadrotor is defined in the ground coordinate system, and the sensor is the attitude and position information of the vehicle body coordinate system, so it is necessary to the body coordinate system to the ground coordinate system. Assuming the body roll angle $\alpha=[\theta, \phi, \psi]$, which $\theta$ is the roll angle, $\phi$ is the pitch angle, $\psi$ is the yaw angle, each angle is defined as shown in figure 2 :

Roll angle $\theta$ : the coordinate system ${ }^{o x y z}$ around the $x$ axis, the $Y$ axis in the $o y z$ plane projection and the angle of the $y$ axis;

Pitch angle $\phi$ : the coordinate system $o x y z$ around the $y$ axis, the $Z$ axis in the $o z x$ plane projection and the angle of the $z$ axis; 
Yaw angle $\psi$ : the coordinate system $o x y z$ around the $z$ axis, the $X$ axis in the $o x y$ plane projection and the angle of the $x$ axis;

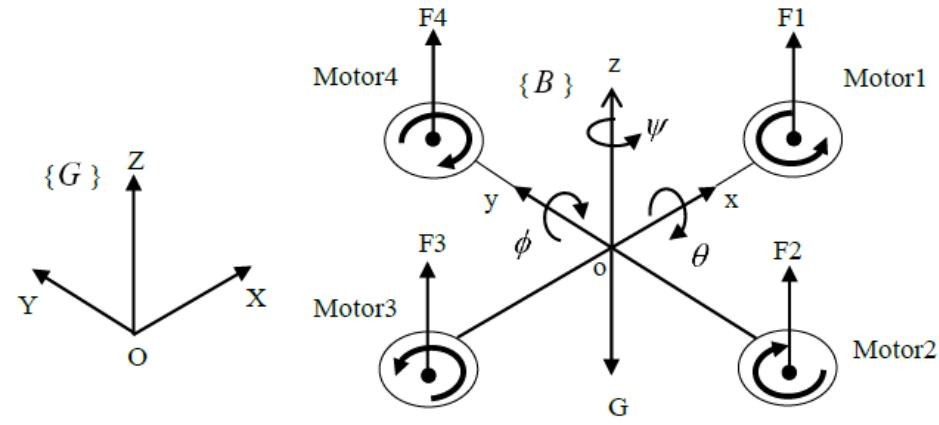

Fig. 1 Structure model of quadrotor

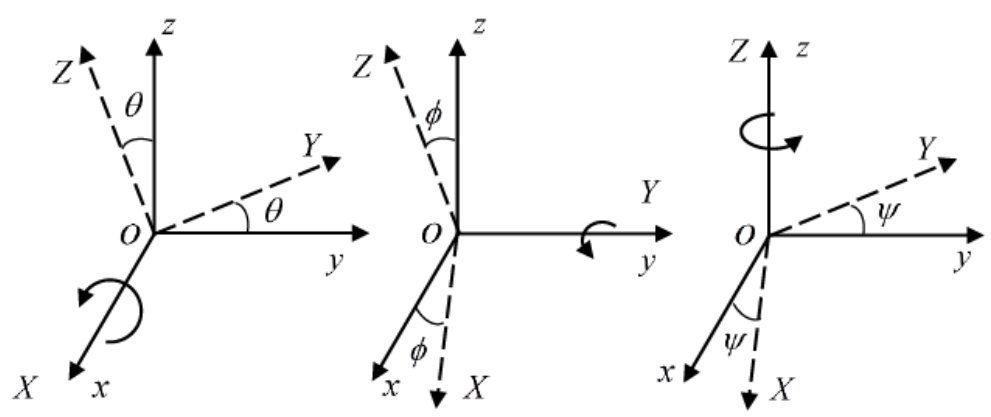

Fig. 2 Schematic diagram of coordinate system transformation

The coordinate transformation matrix of each angle is obtained:

$$
\begin{aligned}
R_{\theta} & =\left[\begin{array}{ccc}
1 & 0 & 0 \\
0 & \cos \theta & -\sin \theta \\
0 & \sin \theta & \cos \theta
\end{array}\right] \\
R_{\phi} & =\left[\begin{array}{ccc}
\cos \phi & 0 & \sin \phi \\
0 & 1 & 0 \\
-\sin \phi & 0 & \cos \phi
\end{array}\right] \\
R_{\psi} & =\left[\begin{array}{ccc}
\cos \psi & -\sin \psi & 0 \\
\sin \psi & \cos \psi & 0 \\
0 & 0 & 1
\end{array}\right]
\end{aligned}
$$

Finally get the whole $3 \mathrm{D}$ space coordinate transformation matrix:

$$
R=R_{\psi} \times R_{\phi} \times R_{\theta}=\left[\begin{array}{ccc}
\cos \psi \cos \phi & \cos \psi \sin \phi \sin \theta-\sin \psi \cos \theta & \cos \psi \sin \phi \cos \theta+\sin \psi \sin \theta \\
\sin \psi \cos \phi & \sin \psi \sin \phi \sin \theta+\cos \psi \cos \theta & \sin \psi \sin \phi \cos \theta-\cos \psi \sin \theta \\
-\sin \phi & \cos \phi \sin \theta & \cos \phi \cos \theta
\end{array}\right]
$$

Vector in the body coordinate system:

$$
A_{B}=\left[x_{B}, y_{B}, z_{B}\right]^{T}
$$

Is converted to the ground coordinate system:

$$
A_{G}=\left[x_{G}, y_{G}, z_{G}\right]^{T}=R \times A_{B}
$$

In order to establish the dynamics model of the quadrotor, the following assumptions are made to the quadrotor [6]:

(1) Quadrotor for uniform and symmetrical rigid body; 
(2) The origin of the ground coordinate system is the geometric center of the quadrotor, and is coincident with the center of mass;

(3) The body is affected by the air resistance can be ignored, regardless of the ground effect;

(4) Four the lift of each rotor is proportional to the square of the rotor speed, and the torque of the rotor is proportional to the square of the rotor speed;

Based on Newton's second law and Euler's formula, the dynamics model equation of quadrotor is established [7]:

$$
F=m \frac{d V}{d t}, M=\frac{d H}{d t}
$$

$F$ is the sum of the external forces of the quadrotor, $m$ for the quality of the quadrotor, $V$ is the speed of the quadrotor, $M$ for the quadrotor by the sum of the torque, $H$ quadrotor relative to the ground coordinates of the moment of momentum.

Set a single propeller speed is $\Omega_{n}$, by the assumption of (4) to each rotor lift is $a \Omega_{n}{ }^{2}$, where $a$ is the proportional coefficient. The total lift of the quadrotor is $U_{1}=a\left(\Omega_{1}^{2}+\Omega_{2}{ }^{2}+\Omega_{3}{ }^{2}+\Omega_{4}{ }^{2}\right)$, the force vector $F_{B}=\left(0,0, U_{1}\right)^{T}$ of the quadrotor in the body coordinate system $B$. By the formula (6) can get the ground coordinate system under the force vector is:

$$
F_{G}=\left[\begin{array}{c}
F_{X} \\
F_{Y} \\
F_{Z}
\end{array}\right]=R \times F_{B}=\left[\begin{array}{c}
(\cos \psi \sin \phi \cos \theta+\sin \psi \sin \theta) U_{1} \\
(\sin \psi \sin \phi \cos \theta-\cos \psi \sin \theta) U_{1} \\
(\cos \phi \cos \theta) U_{1}
\end{array}\right]
$$

The air drag coefficient of the three rotor quadrotor is $X, Y$ and $Z$ in the ground coordinate system. The air resistance coefficient is $K_{X}, K_{Y}, K_{Z}$, and $F=m \frac{d V}{d t}$, according to Newton's second law, the three axis linear equation of the ground coordinate system is established:

$$
\left[\begin{array}{c}
\ddot{x} \\
\ddot{y} \\
\ddot{z}
\end{array}\right]=\left[\begin{array}{c}
\frac{F_{X}-K_{X} \dot{x}}{m} \\
\frac{F_{Y}-K_{Y} \dot{y}}{m} \\
\frac{F_{Z}-K_{Z} \dot{z}-m g}{m}
\end{array}\right]=\left[\begin{array}{c}
\frac{(\cos \psi \sin \phi \cos \theta+\sin \psi \sin \theta) U_{1}-K_{X} \dot{x}}{m} \\
\frac{(\sin \psi \sin \phi \cos \theta-\cos \psi \sin \theta) U_{1}-K_{Y} \dot{y}}{m} \\
\frac{(\cos \phi \cos \theta) U_{1}-K_{Z} \dot{z}}{m}-g
\end{array}\right]
$$

Due to the role of force is mutual, the air will also have a reaction force on the rotor, so the yaw moment is so generated, the torque generated by each rotor is $W_{n}=b \Omega_{n}{ }^{2}$, where $b$ is the reverse torque coefficient. $l$ For the rotor center to the center of the quadrotor to the distance, $U_{2}$ for the rolling moment, $U_{3}$ for the pitching moment, $U_{4}$ for the yaw moment, there are:

$$
\left[\begin{array}{c}
U_{2} \\
U_{3} \\
U_{4}
\end{array}\right]=\left[\begin{array}{c}
l\left(F_{4}-F_{2}\right) \\
l\left(F_{3}-F_{1}\right) \\
W_{4}+W_{2}-W_{3}-W_{1}
\end{array}\right]=\left[\begin{array}{c}
l a\left(\Omega_{4}{ }^{2}-\Omega_{2}{ }^{2}\right) \\
l a\left(\Omega_{3}{ }^{2}-\Omega_{1}{ }^{2}\right) \\
b\left(\Omega_{4}{ }^{2}+\Omega_{2}{ }^{2}-\Omega_{3}{ }^{2}-\Omega_{1}{ }^{2}\right)
\end{array}\right]
$$

Because of the assumption(1), the inertia matrix can be defined as the diagonal matrix $J$, and the three axis angular motion equations can be obtained according to the Euler dynamic equation of rigid body fixed point rotation: 


$$
\left[\begin{array}{c}
\ddot{\theta} \\
\ddot{\phi} \\
\ddot{\psi}
\end{array}\right]=\left[\begin{array}{l}
\frac{U_{2}+\left(J_{y}-J_{z}\right) \dot{\phi} \dot{\psi}}{J_{x}} \\
\frac{U_{3}+\left(J_{z}-J_{x}\right) \dot{\psi} \dot{\theta}}{J_{y}} \\
\frac{U_{4}+\left(J_{x}-J_{y}\right) \dot{\theta} \dot{\phi}}{J_{z}}
\end{array}\right]
$$

Since the aircraft is flying in a windless or indoor environment, the resistance coefficient and the gyro effect can be studied first, so the final mathematical model is simplified as follows

$$
\begin{aligned}
& {\left[\begin{array}{c}
\ddot{x} \\
\ddot{y} \\
\ddot{z}
\end{array}\right]=\left[\begin{array}{c}
(\cos \psi \sin \phi \cos \theta+\sin \psi \sin \theta) \frac{U_{1}}{m} \\
(\sin \psi \sin \phi \cos \theta-\cos \psi \sin \theta) \frac{U_{1}}{m} \\
(\cos \phi \cos \theta) \frac{U_{1}}{m}-g
\end{array}\right]} \\
& {\left[\begin{array}{c}
\ddot{\theta} \\
\ddot{\phi} \\
\ddot{\psi}
\end{array}\right]=\left[\begin{array}{l}
U_{2} / J_{x} \\
U_{3} / J_{y} \\
U_{4} / J_{z}
\end{array}\right]}
\end{aligned}
$$

\section{Controller Design}

The model is nonlinear, and the models used in the design of the controller and the simulation analysis are often linear, so the nonlinear model needs to be linearized. When the quadrotor is in a stable hover, the linear velocity $\dot{x}=0, \dot{y}=0, \dot{z}=0$, in the coordinate system along the coordinate system in the coordinate system, the angular velocity $\dot{\theta}=0, \dot{\phi}=0, \dot{\psi}=0$, f around the rotation of the three axes. Under these conditions, the steady-state values of the control quantities of the four-rotorcraft control system under the conditions of stable suspension of the aircraft are obtained: $U_{1}=m g, U_{2}=U_{3}=U_{4}=0$, the lift of each rotor is $F_{1}=F_{2}=F_{3}=F_{4}=\frac{m g}{4}$.

The linear space model is established for the quadrotor control system using the LPV (Linear parameter varying) method [8]. The approximation method is used and the weak coupling between the force and the moment of the quadrotor is neglected. A set of quadrotor system state space, which set the vertical direction of the aircraft speed $\dot{z}$, roll angular velocity $\dot{\theta}$, pitch angular velocity $\dot{\phi}$, yaw angular velocity $\dot{\psi}$ for the state variable; input for the $U_{1}, U_{2}, U_{3}, U_{4}$ formed by the matrix, The output is the vertical displacement of the aircraft $z$, roll angle $\theta$, pitch angle $\phi$, yaw angle $\psi$. According to the LPV method, the state space equation of the quadrotor system is as follows:

$$
\left\{\begin{array}{l}
\dot{X}=A X+B U \\
Y=C X+D U
\end{array}\right.
$$

Among them $X=\left[\begin{array}{llll}\dot{z} & \dot{\theta} & \dot{\phi} & \dot{\psi}\end{array}\right]^{T}, Y=\left[\begin{array}{llll}z & \theta & \phi & \psi\end{array}\right]^{T}, U=\left[\begin{array}{llll}U_{1} & U_{2} & U_{3} & U_{4}\end{array}\right]^{T}$.

By solving the correlation coefficient matrix, we get the transfer function in the form of state space, as shown in the following formula: 


$$
G_{1}(s)=C(s I-A)^{-1} B+D=\left[\begin{array}{cccc}
\frac{\cos \phi \cos \theta / m}{s} & 0 & 0 & 0 \\
0 & \frac{1 / J_{x}}{s} & 0 & 0 \\
0 & 0 & \frac{1 / J_{y}}{s} & 0 \\
0 & 0 & 0 & \frac{1 / J_{z}}{s}
\end{array}\right]
$$

As the aircraft in a small angle of movement, so there are, so the type can be simplified as:

$$
G_{1}(s)=C(s I-A)^{-1} B+D=\left[\begin{array}{cccc}
\frac{1 / m}{s} & 0 & 0 & 0 \\
0 & \frac{1 / J_{x}}{s} & 0 & 0 \\
0 & 0 & \frac{1 / J_{y}}{s} & 0 \\
0 & 0 & 0 & \frac{1 / J_{z}}{s}
\end{array}\right]
$$

The final linear model of the system is composed of the aircraft body model and the brushless motor model. The brushless motor is described as a first-order inertial element. The transfer function between the motor governor control signal and the rotor-generated lift is:

$$
G_{2}(s)=\frac{d}{0.1 s+1}(15)
$$

$d$ is the proportional coefficient between the control signal of the motor electronic governor and the motor lift. Since the value of $d$ has little effect on the system simulation, it is $d=1$ in order to facilitate the study. Let the relationship between the lift of the quadrotor lift $F$ and $U$ be $G_{3}(s), G_{3}(s)$ is as follows:

$$
G_{3}(s)=\left[\begin{array}{cccc}
\frac{1}{0.1 s+1} & 0 & 0 & 0 \\
0 & \frac{1}{0.1 s+1} & 0 & 0 \\
0 & 0 & \frac{1}{0.1 s+1} & 0 \\
0 & 0 & 0 & \frac{1}{0.1 s+1}
\end{array}\right]
$$

So the final transfer function of the system is:

$$
G(s)=G_{1}(s) G_{3}(s)=\left[\begin{array}{cccc}
\frac{1 / m}{0.1 s^{2}+s} & 0 & 0 & 0 \\
0 & \frac{1 / J_{x}}{0.1 s^{2}+s} & 0 & 0 \\
0 & 0 & \frac{1 / J_{y}}{0.1 s^{2}+s} & 0 \\
0 & 0 & 0 & \frac{1 / J_{z}}{0.1 s^{2}+s}
\end{array}\right]
$$

According to the transfer function shown in equation (19), four controllers are designed for the aircraft.

In this paper, the design of the controller based on the classical PID control method, the control law of the system is designed using closed loop control. The principle of the PID controller is to make the system's output value and expected value to do the difference, and the error signal proportional, integral, differential calculation, the three parts of the sum to get the final control signal [8]. Figure 3 for the PID control schematic. 


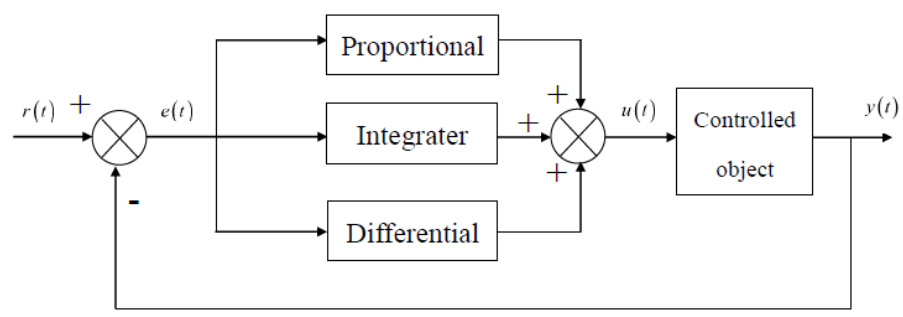

Fig. 3 PID control schematic

The attitude control of the whole control system of the quadrotor is the key of the study. It can be seen from the formula (12) that the attitude change of the aircraft directly affects the linear motion of the aircraft. The parameters of the quadrotor are shown in Table 1 below.

Table 1 Parameters of quadrotor

\begin{tabular}{cccc}
\hline Parameter & Description & Value & Units \\
\hline$m$ & Mass & 0.504 & $\mathrm{~kg}$ \\
$l$ & Distance & 0.25 & $\mathrm{~m}$ \\
$a$ & Proportionality Constant & $3.5 \times 10^{-6}$ & $\mathrm{~N} \cdot \mathrm{S}^{2} \cdot \mathrm{rad}^{-2}$ \\
$b$ & Proportionality Constant & $1.1 \times 10^{-6}$ & $\mathrm{~N} \cdot \mathrm{S}^{2} \cdot \mathrm{rad}^{-2}$ \\
$J_{x}$ & $x$ Inertia & $5.7 \times 10^{-3}$ & $\mathrm{~kg} \bullet \mathrm{m}^{2}$ \\
$J_{y}$ & $y$ Inertia & $5.7 \times 10^{-3}$ & $\mathrm{~kg} \bullet \mathrm{m}^{2}$ \\
$J_{z}$ & $z$ Inertia & $9.2 \times 10^{-3}$ & $\mathrm{~kg} \cdot \mathrm{m}^{2}$ \\
\hline
\end{tabular}

Into the formula (19) to get the actual transfer function of the aircraft as follows:

$$
G(s)=\left[\begin{array}{cccc}
\frac{1.98}{0.1 s^{2}+s} & 0 & 0 & 0 \\
0 & \frac{175.44}{0.1 s^{2}+s} & 0 & 0 \\
0 & 0 & \frac{175.44}{0.1 s^{2}+s} & 0 \\
0 & 0 & 0 & \frac{108.70}{0.1 s^{2}+s}
\end{array}\right]
$$

Figure 4 shows the PID loop structure, according to Figure 4 to build Simulink model and simulation, after debugging the parameters of each channel PID parameters in Table 2, followed by vertical displacement, pitch angle, roll angle and yaw angle, simulation results such as Figure 5.

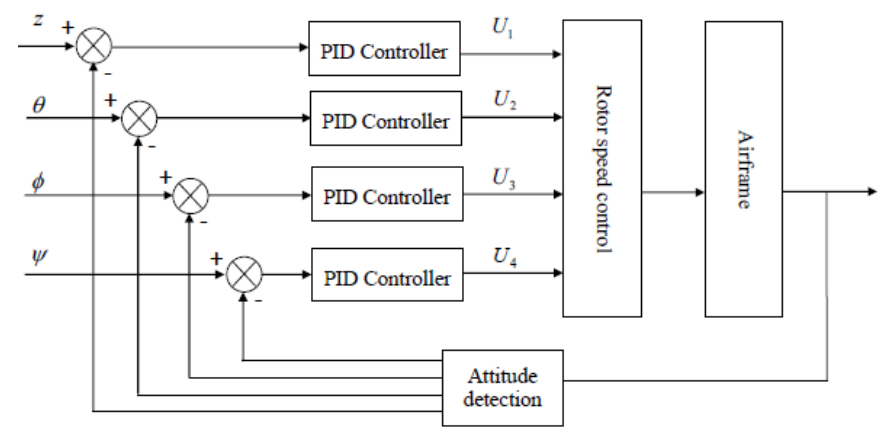

Fig. 4 The structure of attitude PID circuit

Table 2 Parameters of each attitude angle

\begin{tabular}{cccc}
\hline$N$ & $K_{p}$ & $K_{I}$ & $K_{D}$ \\
\hline 1 & 3.2 & 0.01 & 0.21 \\
2 & 0.042 & 0.001 & 0.0035 \\
3 & 0.042 & 0.001 & 0.0035 \\
4 & 0.05 & 0.001 & 0.003 \\
\hline
\end{tabular}



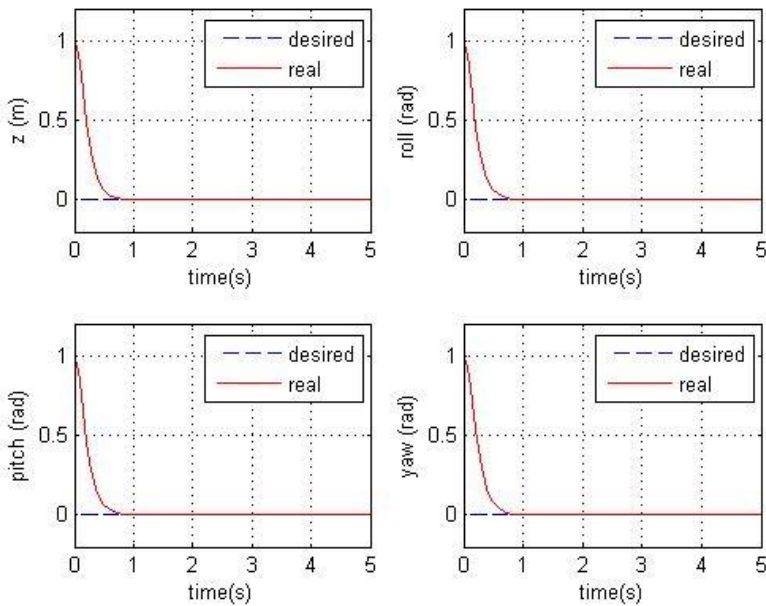

Fig. 5 Each channel step response

It can be seen from the simulation results that the system step response is less than $1 \mathrm{~s}$ when the initial condition of the system is $1 \mathrm{~m}$ and the attitude angle is 1 rad, and there is almost no overshoot and steady-state error. In order to further verify the system's control capabilities, the following simulation was done:
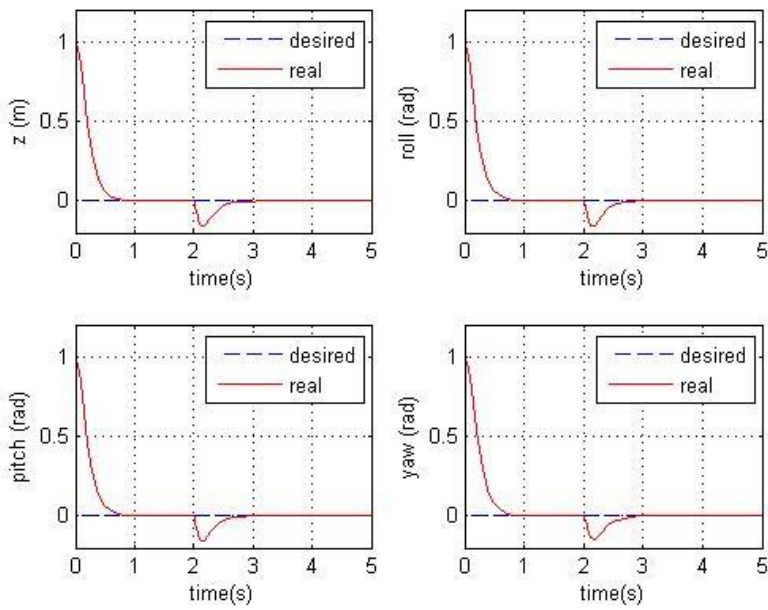

Fig. 6 Anti - jamming response graph

In the simulation of the first $2 \mathrm{~s}$ to the system to add an interference signal, the simulation results from the controller can be within $1 \mathrm{~s}$ to control the aircraft to a steady state, to achieve the desired control objectives. This shows that PID has a significant effect on the quadrotor attitude control.

\section{Conclusion}

The quadrotor is a non-linear, multivariable, strongly coupled underactuated system that is very difficult to control precisely. In this paper, the dynamic model of quadrotor is established by Newton Euler formula. Although the model is nonlinear and cannot be controlled directly by traditional control algorithm, this paper adopts LPV method to linearize the model and then adopt mature PID control algorithm, Using Simulink to build the model and verify the PID on the quadrotor attitude control effectiveness.

\section{Acknowledgements}

This research was supported by Shanghai University of Engineering Science for Graduate Innovation Project No: E3-0903-16-01270. 


\section{References}

[1]. Jintian $\mathrm{Hu}$, HuaiLin Shu. Research on control algorithm of four rotor aircraft based on PID neural network [J]. Automation and Information Engineering, 2015, 01, p.18-22.

[2]. Bowen Nie, Hongxu Ma, Jian Wang, ET. Research status and key technology of Micro Four rotor [J]. Electronics Optics \&amp; Control, 2007, 06, p.113-117.

[3]. Shuqiu Ye, Lin Zhan. Attitude control system of four rotor aircraft based on PID [J]. Computer and modernization, 2015, 05, p.117-120.

[4]. Jun Li, Yuntang Li. Dynamics modeling and PID control of four rotor aircraft [J]. Journal of Liaoning Technical University, 2012, 01, p.114-117.

[5]. Yannong Li, Tinglan Li, Yi Jiang, Jialu Fan. RBF neural network adaptive PID control of four rotor aircraft [J]. Control engineering, 2016, 03, p.378-382.

[6]. Ly Dat Minh. Modeling and control of quadrotor MAV using visionbased measurement [J]. IEEE Trans. Circuits Syst., 2010, 33, p.70-77.

[7]. Zhenglong Deng. Inertial technology [M]. Harbin Institute of Technology press, 2006, p.57-60.

[8]. G. Papageorgiou, K. Glover. Taking Robust LPV Control into Flight on the VACC Harrier. Proceedings of the 39th IEEE conference. On Decision and Control. 2000, p.4560-4564.

[9]. Qingran Dai, Hangyu Li. Attitude control and control of four rotor [J]. Technology Information, 2015, 35, p.33-34. 